\title{
Reduction of chronic post-herniotomy pain and recurrence rate. Use of the anatomical self-gripping ProGrip laparoscopic mesh in TAPP hernia repair. Preliminary results of a prospective study
}

\author{
Pavol Klobusicky ${ }^{1}$, David Hoskovec ${ }^{2}$ \\ ${ }^{1}$ Digestive and Minimal Access Surgery, Helios St. Elisabeth Hospital, Bad Kissingen, Germany \\ ${ }^{2}$ Department of General Digestive Surgery, General Hospital, Prague, Czech Republic
}

Videosurgery Miniinv 2015; 10 (3): 373-381

DOI: $10.5114 /$ wiitm.2015.54222

\begin{abstract}
Introduction: The role of fixation of the mesh is especially important in the endoscopic technique. The fixation of mesh through penetrating techniques using staples, clips or screws is associated with a significantly increased risk of developing a post-herniotomy pain syndrome.

Aim: To demonstrate the safety and efficacy of the self-fixating anatomical Parietex ProGrip laparoscopic mesh (Sofradim Production, Trévoux France) used with laparoscopic transabdominal preperitoneal hernia repair. The incidence of chronic post-herniotomy pain and recurrence rate in the follow-up after 12 months were evaluated.

Material and methods: Data analysis included all patients who underwent inguinal hernia surgery at our Surgical Department within the period from 1.05.2013 to 31.12.2014, who fulfilled the inclusion criteria. Standard surgical technique was used. Data were prospectively entered and subsequently analyzed on the Herniamed platform. Herniamed is an internet-based register in German and English language and includes all data of patients who underwent surgery for some types of hernia.

Results: There were 95 patients enrolled in the group and there were in total 156 inguinal hernias repaired. The mean follow-up was 15.52 months. At the assessment at 1 year mild discomfort in the groin was reported in 2 patients (3.51\%) (1-3 VAS). No recurrence or chronic postoperative pain was reported.

Conclusions: Laparoscopic inguinal hernia repair using the transabdominal preperitoneal technique with implantation of the ProGrip laparoscopic mesh is a fast, effective and reliable method in experienced hands, which according to our results reduces the occurrence of chronic post-operative inguinal pain with simultaneously a low recurrence rate.
\end{abstract}

Key words: laparoscopic inguinal hernia repair, transabdominal preperitoneal, ProGrip laparoscopic mesh, Herniamed.

\section{Introduction}

Inguinal hernia repair is one of the most frequently performed surgical procedures worldwide in general surgery. There are costs of approximately USD 500,000,000 in the United States for inguinal hernia repair plus the costs for medication, sickness leave and lost work performance. This means that from the point of view of costs, an effective and reliable therapy of inguinal hernia is a significant factor that influences efficiency of national systems of public health insurance $[1,2]$. Prosthetic mesh reinforcement is now a routine step in the operative

\section{Address for correspondence}

Pavol Klobusicky, Digestive and Minimal Access Surgery, Helios St. Elisabeth Hospital Bad Kissingen, Kissinger Str. 150,

97688 Bad Kissingen, Germany, phone: +49 9718055001, fax: +49 9718055005, e-mail: pavol.klobusicky@helios-kliniken.de 
management of inguinal hernia, having been shown to significantly reduce the risk of recurrence. The operative technique published by Lichtenstein, which utilizes the open approach and anterior positioning of the implanted mesh, was considered a gold standard in inguinal hernia repairs for a long time. The transabdominal preperitoneal (TAPP) approach in the therapy of inguinal hernia seems to be a suitable alternative to classical open inguinal hernia repair, mainly in the hands of an experienced surgeon [3]. According to several studies comparing open and laparoscopic hernia repair, laparoscopic inguinal hernia repair is a suitable alternative primarily in cases of recurrent hernias and bilateral hernias $[1,4,5]$. The Lichtenstein procedure is advantageous mainly due to its low cost, the possibility of an outpatient procedure, the use of local anesthesia and furthermore its relatively good results, mainly with regard to the number of recurrences [2]. On the other hand, it should be noted that it is an open procedure, necessitating open dissection of the groin with implantation of the mesh in the anterior position, which results in longer post-operative recovery and significantly higher incidence of pain in the groin, particularly in the first 3 months after surgery, and also leads to a higher incidence of chronic post-operative inguinal pain (CPIP) [2]. Laparoscopic inguinal hernia repair offers the possibility of gentle dissection with posterior implantation of the mesh and the possibility of minimally invasive fixation of the implanted mesh [2, 6-8]. Postoperative pain, however, remains a significant clinical problem, which is often attributed to the characteristics of the mesh and/or the method of fixation used [6]. Several prosthetic materials intended for implantation in the groin have been introduced on the market; their safety and efficacy have been demonstrated by several studies $[2,8]$. Originally it was necessary and recommended to stabilize the implant at the site of implantation with additional invasive fixation in the form of spirals, clips, screws, etc. These implants in inappropriate locations often were the cause of CPIP and also relatively dramatically increased the costs for that surgery. Current trends in TAPP repair are to use staples or fibrin glue, the latter now known to reduce postoperative pain, complications, and time to resumption of normal activities [6]. The option to use self-fixation meshes in inguinal hernia repair was verified in an animal experiment as safe, well tolerated and simple to perform, with good macroscopic and microscopic integration to the abdominal wall $[9,10]$. Subsequently, self-fixation meshes that were originally intended for open implantation in inguinal hernia repairs using the Lichtenstein technique were introduced to clinical practice. Good tolerance, shortened operation time and significantly improved quality of life (QoL) were demonstrated in the questionnaires filled out by patients, while there was an unchanged rate of recurrences $[11,12]$. Parietex ProGrip laparoscopic mesh (produced by Covidien, Sofradim Production, Covidien, France) was the first self-fixating mesh introduced to the market intended for endoscopic/laparoscopic implantation. Usage and good efficiency with unchanged recurrence rate and unchanged frequency of CPIP were demonstrated in a retrospective study also in the case of laparoscopic TAPP inguinal hernia repair [2]. Laparoscopic inguinal hernia repair was introduced to practice in 1991 and includes the standard transumbilical approach with dissection of parietal peritoneum, treatment of the hernia sac and posterior positioning of implanted material to cover the defect. The surface of the mesh is used for fixation of the mesh to the required position; after contact with tissue and combination with fine mechanical pressure, a solid connection is created, which prevents secondary shift or migration of the mesh, so that there is no need for further fixation of the mesh. The mechanism of gripping is as follows: The microgrips integrate into the tissue $0.5 \mathrm{~mm}$ below the lower rim and provide stronger tissue incorporation at 5 days than fixation with staples [10]. Fixation is, therefore, greatly facilitated, without requiring sutures that can penetrate underlying tissues and damage nerves. The goal of this study, based on single-centre prospective collection of data from the Internet-based multicenter Herniamed register, is to demonstrate the safety and efficacy of the use of Parietex ProGrip laparoscopic mesh in standardized laparoscopic inguinal hernia repair with the TAPP technique. Although this technique of inguinal hernia repair has been in standard use in our workplace since 2008 and more than 500 patients have already been operated on, but using other (older) types of mesh, we only evaluated data from the 95 patients who were operated on at our workplace with the standardized technique first described by Bittner et al. [1] with implantation of an anatomical self-gripping Parietex ProGrip laparoscopic mesh, and all data were prospectively collected and stored using the Herniamed study. In 
total there were 156 inguinal hernias treated in this way. All operations were performed by two surgeons with advanced experience with this type of operation technique. We performed a statistical analysis of the data and evaluated all patients at the first day (D1), first month (M1) and first year (Y1) after surgery. Analysis of data was performed focusing on post-operative complications, postoperative pain, CPIP and recurrence rates. At the same time, analysis of risk factors was performed and their effect on the possible recurrence rate was assessed.

Individual inclusion and exclusion criteria are given in Table I.

\section{Aim}

The aim of the study was to demonstrate the safety and efficacy of the self-fixating anatomical Parietex ProGrip laparoscopic mesh (Sofradim Production, Trévoux France) used with laparoscopic transabdominal preperitoneal hernia repair. The incidence of chronic post-herniotomy pain and recurrence rate in the follow-up after 12 months were evaluated.

\section{Material and methods}

Data analysis encompassed all patients who underwent inguinal hernia surgery at the Surgical Department in Bad Kissingen, Bavaria, Germany within the period from May 1, 2013 to December 31, 2014, who fulfilled the inclusion criteria. A direct control group (e.g., a group of patients operated on using TAPP technique with staple fixation of mesh) was not established, as the tested hypothesis focused on a favorable change in the QoL in patients subjected to using a Parietex ProGrip laparoscopic mesh, not the comparison of those methods. A standard surgical technique was used. This technique was first described by Bittner et al. [1]. There were two surgeons with experience in this particular technique performing the surgery. Data were entered and subsequently analyzed on the Herniamed platform.

\section{Database}

Herniamed is Europe's biggest Internet-based multicentre register in German and English for prospective collection of data that includes all data of patients who have undergone surgery for some type of hernia. All relevant patient data are collected via the Internet (comorbidity, previous operation, type and size of mesh, specification of surgical procedure,
Table I. Inclusion and exclusion criteria

\begin{tabular}{|l|}
\hline Inclusion criteria: \\
\hline Age $>18$ years \\
\hline Patients operated on with TAPP technique for inguinal \\
hernia using Parietex ProGrip laparoscopic mesh, without \\
additional fixation, between May 2013 and December \\
2014 \\
\hline $\begin{array}{l}\text { Patients successfully and completely documented in } \\
\text { online database Herniamed }\end{array}$ \\
\hline Exclusion criteria: \\
\hline Age $<18$ years \\
\hline Scrotal/large hernia with defect $>4$ cm \\
Patients with incarcerated hernia needing emergency \\
surgery \\
Patients with ASA IV or patients who could not be operat- \\
ed on under general anesthesia for other reasons \\
\hline
\end{tabular}

used materials, peri- and post-operative complications, gender, body mass index (BMI), and risk factors in the patient) [13].

\section{Data collection}

Surgical risk was evaluated according to the American Society of Anesthesiologists (ASA). Type of hernia, size of hernia opening, incarceration, perioperative complications, materials used, supplementary procedures and duration of surgical procedure were classified during the surgery. Complications and post-operative pain were evaluated based on the visual analogue scale (VAS) 0-10, where 0 indicates no pain, 1-3 mild pain (not evaluated as pain), 4-6 moderate pain and 7-10 severe pain. Post-operative pain was evaluated on D1 and M1 after the operation by examination of the patient and at $\mathrm{Y} 1$ after the operation using an automatic questionnaire of the Herniamed database. Cases with suspicion of recurrence and cases with possible CPIP were also examined clinically by the surgeon. Postoperative complications were defined as significant/major, which required prolongation of hospitalization, and less significant/minor, which had no greater effect on the post-operative course in the patient.

As recurrence was evaluated, ultrasound and magnetic resonance imaging (MRI) demonstrated recurrent hernia.

Follow-up took place after 1 year from surgery in the form of a standardized questionnaire according to Herniamed [13]. 


\section{Mesh}

Parietex ProGrip laparoscopic mesh is a self-gripping composite mesh, consisting of a monofilament polyethylene terephthalate mesh (PET), covered by a resorbable layer of microgrips made of polylactide acide (PLA) combined with a segmental covering of fast resorbing film composition (70\% collagen, $30 \%$ glycerol). The weight of the mesh before $a b-$ sorption is $82 \mathrm{~g} / \mathrm{m}^{2}$ and the weight after absorption is $49 \mathrm{~g} / \mathrm{m}^{2}$, with the pore size $(\mathrm{mm}) 1.8 \times 1.8$ (macroporous). According to the manufacturer, the time for degradation of the quickly absorbing layer is $<1$ day and that for the layer with microgrips $>18$ months.

\section{Operation}

Standard intravenous anesthesia with orotracheal intubation was done, which enables the Trendelenburg position. The operation was performed with a standard operating technique in the Trendelenburg position using three trocars, with the $12 \mathrm{~mm}$ trocar

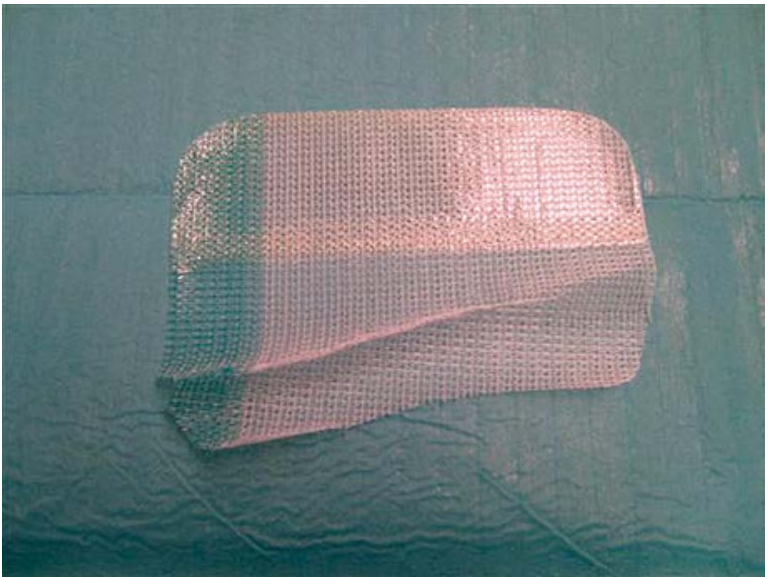

Photo 1. Anatomical self gripping Parietex ProGrip laparoscopic mesh

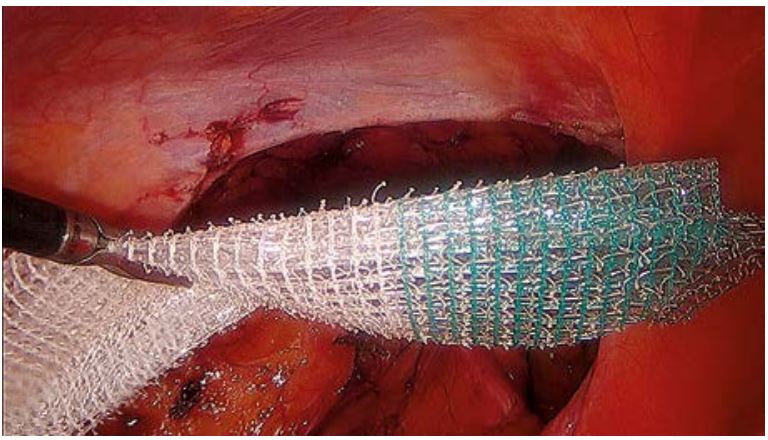

Photo 2. Folded mesh above the umbilicus and the $5 \mathrm{~mm}$ and $12 \mathrm{~mm}$ trocars at the level of the umbilicus in the left and right medioclavicular lines. Capnoperitoneum was established under a pressure of $12 \mathrm{~mm} \mathrm{Hg}$ and a $30^{\circ}$ optic was used. We used the technique described by Bittner [1]. After accessing the inguinal region, dissection of the parietal peritoneum on the affected side was performed in the direction from the anterior superior iliac spine up to the plica umbilicalis medialis on the same side. During dissection, gonadal vessels, vas deferens or the round ligament (in women), Cooper's ligament and the posterior fascia of the rectus abdominis muscle were visualized and prepared. After ending dissection in the groin, we introduced the prepared implant with size $15 \times$ $10 \mathrm{~cm}$ through the $12 \mathrm{~mm}$ trocar. The implant was spread out in the abdominal cavity and placed in the groin in order to cover the hernia opening by at least $2-3 \mathrm{~cm}$ in all directions and also to cover other preformed weaker parts in the groin. Fixation to the abdominal wall was accomplished by gently pressing the implant against the abdominal wall using a surgical instrument, but most effectively with a tampon. Fixation was based on a mechanical effect involving the adherence of grips to tissue. The reconstruction of the parietal peritoneum followed, with continuous absorbable suture. In case of a bilateral hernia, treatment is done with the same method and technique, covering both meshes in the prevesical space without the need to change the location of trocars. To reduce urethral manipulation in an effort to minimize post-operative urinary retention, patients void preoperatively and a Foley catheter is not routinely placed. No antibiotic prophylaxis is routinely administered during the surgery (Photos 1-4).

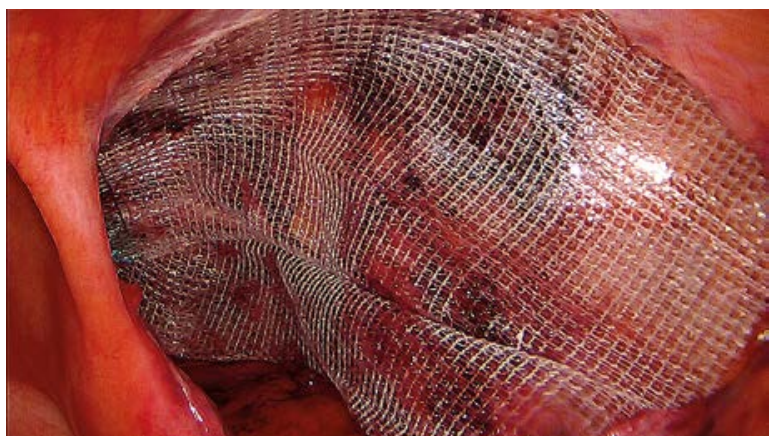

Photo 3. Terminal mesh position after implantation 


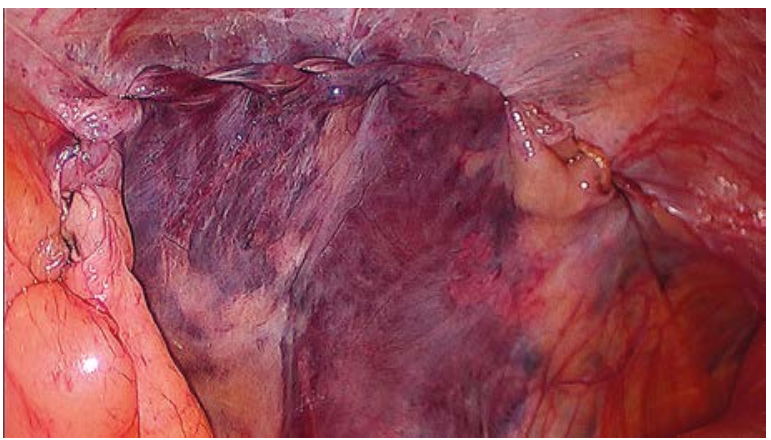

Photo 4. Running suture of peritoneum

\section{Results}

In total, 951 patients were listed in the prospective, completely processed documentation on the Herniamed platform. In total, 156 hernias were operated on. The demographic data and preoperative data are summarized in Table II. Perioperative data and information about the size and localization of hernia are provided in Table III. There were 95 patients enrolled in the presented group and 156 inguinal hernias were repaired. From the demographic point of view there were $85(89.47 \%)$ men and 10 (10.53\%) women out of a total of 95 , with the clear dominance of $50-60$ years (mean age: $57.4 \pm 12.1$ years) in men and 50-60 years (mean age: $54.4 \pm 12.8$ years) in women. This was a heterogeneous group of patients simulating an average, non-selected group of patients undergoing inguinal hernia repair. Representation of females in the group was around onetenth $(10 / 95,10.53 \%)$, while these were mostly bilateral hernias. One-half of the patients were classified as ASA II (49/95, 51.57\%), while 8 (8.42\%) patients were classified as ASA III. Up to 49 (51.57\%) patients had had some abdominal surgery in the past. Most frequently there was a history of an open hernia repair (26 patients, $27.37 \%$ ) and open appendectomy (17 patients, $17.89 \%$ ). The duration of hospital stay varied between unilateral and bilateral disease, while the average duration of hospital stay was 1.66 days for unilateral operation and 1.90 days for bilateral hernia. The average operation time was $64 \mathrm{~min}$, while time taken for bilateral hernias was $67 \mathrm{~min}$, which is not much different from other comparable published papers $[2,14]$. It should be emphasized that operating time for medial hernias is significantly shorter than for lateral hernias and also recurrent hernias, which is in accord with other authors [2]. The majority of operated hernias, i.e., 143 (91.67\%)
Table II. Demographic and preoperative data $(n=95)$

\begin{tabular}{|lcc|}
\hline $\begin{array}{l}\text { Demographic and preoperative } \\
\text { data }\end{array}$ & $\begin{array}{c}\text { Number } \\
\text { of patients }\end{array}$ & $\begin{array}{c}\text { Percent } \\
\text { or range }\end{array}$ \\
\hline \begin{tabular}{l} 
Gender: \\
\hline Male
\end{tabular} & 85 & 89.47 \\
\hline Female & 10 & 10.53 \\
\hline BMI, mean [kg/m²] & 28.2 & $19.9-43.3$ \\
\hline \begin{tabular}{l} 
ASA class: \\
\hline I
\end{tabular} & 38 & 40.00 \\
\hline II & 49 & 51.58 \\
\hline $\begin{array}{l}\text { IV } \\
\text { IV comorbidity): }\end{array}$ & 8 & 8.42 \\
\hline \begin{tabular}{l} 
COPD/bronchial asthma \\
\hline $\begin{array}{l}\text { Diabetes mellitus } \\
\text { wation of thrombocytes, }\end{array}$
\end{tabular} & 0 & 0.00 \\
\hline $\begin{array}{l}\text { Aortal aneurysm } \\
\text { in noumarin derivates (Quick/INR }\end{array}$ & 0 & 23.16 \\
\hline \begin{tabular}{l} 
Immunosuppression \\
\hline Cortisone
\end{tabular} & 13 & 1.05 \\
\hline $\begin{array}{l}\text { Nicotine overuse } \\
\text { Coagulation disorders }\end{array}$ & 0 & 4.21 \\
\hline
\end{tabular}

hernias, had hernia openings of moderate and large sizes (types 2 and $3,>1.5 \mathrm{~cm}$ and $<4 \mathrm{~cm}$ ); medial hernias were predominant on both sides. During the surgery, no complications were demonstrated; post-operative complications were evaluated as significant in relation to previous surgical procedures (major) and as less significant (minor), which did not influence the duration of hospitalization, and these were managed conservatively on an outpatient basis.

A summary of complications is presented in Table IV.

We reported 1 case of minor bleeding (abdominal wall) and there was only 1 case of severe trocar side bleeding. This case was treated with the laparoscop- 
Table III. Perioperative data and hernia details during operation $(n=95)$

\begin{tabular}{|c|c|c|}
\hline \multirow{2}{*}{$\begin{array}{l}\text { Perioperative data } \\
\text { Elective surgery }\end{array}$} & \multicolumn{2}{|c|}{ Results } \\
\hline & 95 & $100 \%$ \\
\hline Number of hernias: & 156 & \\
\hline Unilateral hernia: & 34 & $35.79 \%$ \\
\hline Right sided & 20 & \\
\hline Left sided & 14 & \\
\hline Bilateral hernias & 61 & $64.21 \%$ \\
\hline Recurrent hernias & 32 & $20.51 \%$ \\
\hline Average time of surgery [min]: & 64 & $27-127$ \\
\hline Unilateral hernia & 58 & $27-88$ \\
\hline Bilateral hernia & 67 & $45-127$ \\
\hline EHS classification: & Right sided hernia 51.92\% & Left sided hernia $48.08 \%$ \\
\hline Medial & $50(61.73 \%)$ & $41(54.67 \%)$ \\
\hline Lateral & $27(33.33 \%)$ & $32(42.67 \%)$ \\
\hline Femoral & $2(2.47 \%)$ & $1(1.33 \%)$ \\
\hline Scrotal & $2(2.47 \%)$ & $1(1.33 \%)$ \\
\hline Size of hernia opening: & Right sided & Left sided \\
\hline$<1.5 \mathrm{~cm}, 13(8.33 \%)$ & 9 & 4 \\
\hline $1.5-3 \mathrm{~cm}, 105$ (67.31\%) & 55 & 50 \\
\hline$>3 \mathrm{~cm}, 38(24.36 \%)$ & 17 & 21 \\
\hline
\end{tabular}

Table IV. Complications

\begin{tabular}{|c|c|c|}
\hline Postoperative major complications related to the surgical procedure & $n=95$ & $1.05 \%$ \\
\hline Postoperative hemorrhage in the area of the groin extraperitoneally & 0 & $0.00 \%$ \\
\hline Secondary intraperitoneal bleeding on trocar side & 1 & $1.05 \%$ \\
\hline Postoperative mechanical ileus & 0 & $0.00 \%$ \\
\hline Postoperative minor complications related to the surgical procedure & $n=95$ & $2.11 \%$ \\
\hline Seroma & 1 & $1.05 \%$ \\
\hline Hematoma in abdominal wall & 1 & $1.05 \%$ \\
\hline Hematuria & 0 & $0.00 \%$ \\
\hline
\end{tabular}

ic technique; there was no need of mesh application. Long-term follow-up, recurrence and chronic pain evaluation are summarized as follows. Standard long-term follow-up after 12 months was done in 57 $(60.00 \%)$ patients; in the rest of the patients it was performed at the close of the study, although at least
6 months after the operation. The mean follow-up was at 15.52 months. Long-term follow-up was performed with the standard questionnaire based on the Herniamed platform. There were questions regarding post-operative complications, post-operative pain longer than 3 months according to the VAS, 
Table V. Patient-reported pain using VAS scale prior to surgery and each follow-up evaluation

\begin{tabular}{|lcccc|}
\hline VAS scale & $\begin{array}{c}\text { Before surgery } \\
(N=95), n(\%)\end{array}$ & $\begin{array}{c}\text { D1 }(N=95), \\
n(\%)\end{array}$ & $\begin{array}{c}\text { M1 }(N=95), \\
n(\%)\end{array}$ & $\begin{array}{c}\text { Y1 }(N=57 / 95,60 \%), \\
n(\%)\end{array}$ \\
\hline None (VAS 0) & $34(35.79)$ & $14(14.74)$ & $82(86.32)$ & $55(96.49)$ \\
\hline Mild (VAS 1-3) & $45(47.37)$ & $76(80.00)$ & $13(13.68)$ & $2(3.51)$ \\
\hline Moderate (VAS 4-6) & $16(16.84)$ & $5(5.26)$ & $1(1.05)$ & $0(0.00)$ \\
\hline Severe (VAS > 6) & $0(0.00)$ & $0(0.00)$ & $0(0.00)$ & $0(0.00)$ \\
\hline
\end{tabular}

type of pain (resting, during movements), need of medicamentous therapy and, possibly, recurrence or reoperation. Post-operative pain of moderate degree (4-6 VAS) lasting over 3 months in the groin was reported in 1 case (1.05\%). At the 1 -year assessment, mild discomfort was reported in the groin in 2 from 57 (3.51\%) patients; it had no effect on any patient's usual activity and did not require any therapy (1-3 VAS). After 1 year, there was no patient with CPIP reported. Pain analysis using VAS scores is presented in Table $V$, and the analysis of chronic pain in Table VI. There was no recurrence of inguinal hernia confirmed in the followed-up group.

\section{Discussion}

Laparoscopic inguinal hernia repair has become one of the standard methods to treat inguinal hernia, mainly in cases of recurrent hernias, bilateral hernia and femoral hernia [15]. There have been many studies and meta-analyses comparing laparoscopic endoscopic and open inguinal hernia repairs showing comparable long-term results focusing on the recurrences, post-operative pain and QoL of patients $[1,4,15]$.

Mild differences were reported in the occurrence of chronic post-operative pain [16]. Although there have been studies that found a higher recurrence rate in laparoscopic repairs than in inguinal hernia repairs, it may be more a question of the experience of the operating surgeon than of a real failure of surgical technique [7]. One of the remaining problems in the field of laparoscopic inguinal hernia repair is the problem of fixation or non-fixation of prosthetic material. It has been shown that traumatic fixation of the mesh increases the possibility for the development of CPIP $[17,18]$. This is why atraumatic fixation of prosthetic material is recommended $[17,18]$. For example, in case of endoscopic totally extraperitoneal (TEP) technique, it has been shown that it is possible to withdraw from traumatic fixation of the
Table VI. Analysis of chronic pain after 1 year (Y1) $(n=57(57 / 95))$

\begin{tabular}{|lc|}
\hline VAS scale & Y1, $n(\%)$ \\
\hline None (VAS 0) & $55(96.49)$ \\
\hline Mild (VAS 1-3) & $2(3.51)$ \\
\hline Moderate (VAS 4-6) & $0(0.00)$ \\
\hline Severe (VAS > 6) & $0(0.00)$ \\
\hline
\end{tabular}

implant without increasing the risk of recurrence $[4,19]$. An alternative to atraumatic fixation with tissue glue is the use of the so-called self-fixation mesh, which enables sufficient implant fixation to the abdominal wall, provided by a specially adjusted surface without the need of additional fixation [2, 3]. Birk et al. presented the first larger, retrospective study focusing on the use of the Parietex ProGrip implant, with excellent results in terms of CPIP and recurrence rate [2].

Intraoperatively we have not seen any injury or relevant complication. Post-operative complications which we recorded included only 1 severe hemorrhage at the trocar side. This case was treated with laparoscopy, and there was no need to remove the implant. We recorded post-operative complications in $3(3.15 \%)$ patients in our study, while only 1 (1.05\%) patient developed a more significant complication. The authors considered any previous abdominal surgery, mainly infra-umbilical surgery, which significantly prolongs duration of surgery, as a partial risk factor.

The primary goal of successful inguinal hernia repair is reconstruction of the groin without recurrence and without development of chronic CPIP or reduced QoL.

\section{Postoperative pain}

It has been demonstrated that the use of VAS for the quantification of post-operative pain is 
simple and satisfactory in hernia surgery [20]. In our study, we observed chronic pain lasting more than 3 months in 1 (1.05\%) patient. In this case it was unilateral pain after bilateral repair, which subsided after conservative therapy within $\mathrm{Y} 1$ after the operation and in the $\mathrm{Y} 1$ follow-up was not demonstrated anymore. No patient with CPIP was reported. This positive result of CPIP occurring is promising but generally in accord with other authors [2]. Mild discomfort in the groin, which could not be evaluated as pain, was reported in $1.05 \%$ of patients. It was not limiting in daily life and did not require any therapy. Surprisingly, there was no post-operative pain reported even on D1 (VAS =0) in $14(14.74 \%)$ patients. Also interesting is the relationship of preoperative and post-operative pain, when preoperative pain developed as a negative predictive factor of post-operative pain. This fact confirms the work of Aasvang et al., who in 1 paper defined the negative predictive factors of chronic post-operative pain [21].

\section{Recurrence}

There was no case of recurrent hernia reported. In our opinion, more time is needed for assessment of hernia recurrence. So, the study is in progress with a follow-up after 5 and 10 years, which is according to the protocol of multicentre prospective collection of data of the Herniamed study. The follow-up protocol after 5 and 10 years is the same as the follow-up protocol in the present study after 1 year.

\section{Mesh}

In this study the Parietex ProGrip laparoscopic implant was used. The standard size of the mesh was used $-15 \times 10 \mathrm{~cm}$. There is no need for any special instrumental or additional equipment for implantation of the mesh, and also there is no need to use additional protective plastic foil (as required by the older Parietex ProGrip mesh) as the mesh is provided with an additional surface layer, which prevents the sticking together of the mesh surface. The mesh is folded according to the instructions, and after placement in the abdomen it is unrolled without problems. The fixation is done by the self-fixing surface. In the case of the older Parietex ProGrip implant, it is necessary to roll the mesh together with an artificial plastic foil, which is part of the package, in such a way that sticking together of the mesh surface is avoided as the mesh surface is provided with small self-fixing micro-grips. Intraperitoneally the mesh is unrolled, fixed to the required position, and the plastic foil is removed from the peritoneal cavity. Handling with the new Parietex ProGrip laparoscopic mesh is much easier and practicable in daily routine.

\section{Seroma}

A frequently reported post-operative complication of TAPP inguinal hernia repair is seroma. It was documented in only 1 (1.05\%) case in our group, which is attributed to the consequent suture of the hernia opening with fixation of transversal fascia in direct inguinal hernias in those cases. In our series, we observed no wound infection or mesh infection. Prophylactic administration of antibiotics was demonstrated as unjustified apart from cases with clearly increased risk [4]. Several studies have demonstrated that non-fixation inguinal hernia repair is generally more cost-effective than fixating inguinal hernia repair [14]. The goal of our study was not to analyze the costs, but it is logical that in spite of the slightly higher price of the self-fixation implant, the total cost for the implant and the fixation medium is significantly higher. It may be argued that the costs for open inguinal hernia repair are significantly lower, mainly based on the costs for laparoscopic instrumentation and equipment. However, making this comparison was not the goal of the work. While respecting the current knowledge and certainly the shorter recovery time, better QoL and lower frequency of CPIP, and perhaps also the lower recurrence rate, the use of laparoscopic/endoscopic inguinal hernia repair is preferable also with obviously higher material costs [2].

\section{Conclusions}

According to our experience, considering that this technique of inguinal hernia repair with the self-fixation mesh has been in standard use since 2008 and that more than 500 patients (including other types of self-fixating mesh) have undergone the operation, it is a suitable and effective alternative for all laparoscopic surgeons. The legitimacy of the use of the self-fixation mesh was demonstrated in an experiment [10], in open inguinal hernia repair [22], TEP technique $[19,23]$ and in TAPP technique $[2,14]$. Our study demonstrates that laparoscopic inguinal hernia repair using the TAPP technique with 
implantation of a new Parietex ProGrip laparoscopic self-fixation mesh is a fast, effective, reliable and economically advantageous method in experienced hands, which combines the advantages of laparoscopic approach with simple and practical implantation of the self-fixation mesh, which according to our results reduces the occurrence of CPIP and the recurrence rate. The multicentre prospective study Herniamed is in progress with follow-up after 5 and 10 years, which is according to the protocol of collection of data of the study. The study offers continuous results and will be enlarged and published again after evaluation of the mentioned follow-up. Based on preliminary results of the submitted study, it is possible to recommend the technique mentioned above to surgeons experienced in laparoscopic TAPP (probably also TEP) of inguinal hernia repair, with the use of a self-fixation mesh, as an advantageous, and possibly in future also standard, combination of surgical technique and an implant.

\section{Conflict of interest}

The authors declare no conflict of interest.

\section{References}

1. Bittner R, Schwarz J. Inguinal hernia repair: current surgical techniques. Langenbecks Arch Surg 2011; 397: 271-82.

2. Birk D, Hess S, Garcia-Pardo C. Low recurrence rate and low chronic pain associated with inguinal hernia repair by laparoscopic placement of Parietex ProGrip ${ }^{\text {TM }}$ mesh: clinical out comes of 220 hernias with mean follow-up at 23 months. Hernia 2013; 17: 313-20.

3. Agresta F, Torchiaro M, Tordin C. Laparoscopic transabdominal inguinal hernia repair in community hospital settings: a general surgeon's last 10 years experience. Hernia 2014; 18: 745-50.

4. Miserez M, Peeters E, Aufenacker T, et al. Update with level 1 studies of the European Hernia Society guidelines on the treatment of inguinal hernia in adult patients. Hernia 2014; 18: 151-63.

5. Bittner R, Arregui ME, Bisgaard T, et al. Guidelines for laparoscopic (TAPP) and endoscopic (TEP) treatment of inguinal hernia [International Endohernia Society (IEHS)]. Sur Endosc 2011; 25: 2773-843

6. Kosai N, Sutton PA, Evans J, Varghese J. Laparoscopic preperitoneal mesh repair using a novel self-adhesive mesh. J Minim Access Surg 2011; 7: 192-4.

7. Kukleta JF, Freytag C, Weber M. Efficiency and safety of mesh fixation in laparoscopic inguinal hernia repair using $n$-butyl cyanoacrylate: long-term biocompatibility in over 1,300 mesh fixations. Hernia 2012; 16: 153-62.

8. Fortelny RH, Schwab R, Glaser KS, et al. The assessment of quality of life in a trial on light weight mesh fixation with fibrin sealant in transabdominal preperitoneal hernia repair. Hernia 2008; 12: 499-505.
9. Champault G, Polliand C, Dufour F, et al. A "self adhering" prosthesis for hernia repair: experitmental study. Hernia 2009; 13: 49-52.

10. Hollinsky C, Kolbe T, Walter I, et al. Comparison of a new self-gripping mesh with other fixation methods for laparoscopic hernia repair in a rat model. J Am Coll Surg 2009; 208: 1107-14.

11. Jorgensen LN, Sommer T, Assaadzadeh S, et al.; Danish Multicentre DANGRIP Study Group. Randomized clinical trial of self-gripping mesh versus sutured mesh for Lichtenstein hernia repair. Br J Surg 2013; 100: 474-81.

12. Anadol AZ, Akin M, Kurukahvecioglu O, et al. A prospective comparative study of the efficacy of convential Lichtenstein versus self-adhesive mesh repair for inguinal hernia. Surg Today 2011; 41: 1498-503.

13. Stechemesser B, Jacob DA, Schug-Paß C, Köckerling F. Herniamed: an internet-based registry for outcome research in hernia surgery. Hernia 2012; 16: 269-76.

14. Fumagalli Romario U, Puccetti F, Elmore U, et al. Self gripping mesh versus staple fixation in laparoscopic inguinal hernia repair: a prospective comparison. Surg Endosc 2013; 27: 1798-802.

15. Simons MP, Aufenacker T, Bay-Nielsen M, et al. European Hernia Society guidelines on the treatment of inguinal hernia in adult patients. Hernia 2009; 13: 343-403.

16. Quyn AJ, Weatherhead KM, Daniel T. Chronic pain after open inguinal hernia surgery: suture fixation versus self-adhesive mesh repair. Langebecks Arch Surg 2012; 397: 1215-8.

17. Taylor C, Layani L, Liew V, et al. Laparoscopic inguinal hernia repair without mesh fixation, early result of a large randomised clinical trial. Surg Endosc 2008; 22: 757-62.

18. Brügger L, Bloesch $M$, Ipaktchi R, et al. Objective hypoesthesia and pain after transabdominal preperitoneal hernioplasty: a prospective, randomized study comparing tissue adhesive versus spiral tacks. Surg Endosc 2012; 26: 1079-85.

19. Bresnahan E, Bates A, Wu A, et al. The use of self-gripping (ProGripTM) mesh during laparoscopic total extraperitoneal (TEP) inguinal hernia repair: a prospective feasibility and long-term outcomes study. Surg Endosc 2015; 29: 2690-6.

20. McCarthy M Jr, Chang CH, Pickard AS, et al. Visual analog scales for assessing surgical pain. J Am Coll Surg 2005; 201: 245-52.

21. Aasvang EK, Gmaehle E, Hansen JB, et al. Predictive risk factors for persistent postherniotomy pain. Anesthesiology 2010; 112: 957-69.

22. Kingsnorth A, Gingell-Littlejohn M, Nienhuijs S, et al. Randomized controlled multicenter international clinical trial of self-gripping Parietex ${ }^{\mathrm{TM}}$ ProGrip ${ }^{\mathrm{TM}}$ polyester mesh versus lightweight polypropylene mesh in open inguinal hernia repair: interim results at 3 months. Hernia 2012; 16: 287-94.

23. Teng YJ, Pan SM, Liu YL, et al. A meta-analysis of randomized controlled trials of fixation versus non fixation of mesh in laparoscopic total extraperitoneal inguinal hernia repair. Surg Endosc 2011; 25: 2849-58.

Received: 20.07.2015, accepted: 19.08.2015. 